\title{
Interacción y Movimiento en el Análisis Visual de Proyectos Arquitectónicos Académicos
}

\author{
Interaction and Motion in the Visual Analysis of Academic Architectural Projects
}

\author{
> Felipe Etchegaray Heidrich \\ Universidade Federal de Pelotas, Brasil \\ felipeheidrich@gmail.com
}

\author{
> Ernesto Redondo \\ Universidad Politécnica de Cataluña, España \\ ernesto.redondo@upc.edu
}

\begin{abstract}
This paper proposes two methods for the development of graphical representations with interactivity and motion and describes research done with some architecture students from a school in Brazil to first investigate the possibility of using the proposed representations. Data from this study indicate the possibility of the introduction, regarding the use and manipulation by the students of the two types of representation proposed for visual analysis of academic architectural projects.
\end{abstract}

Keywords: Architectural Representation, Interaction, Motion, Visual Analysis.

\section{Introducción}

El presente estudio es parte de una investigación que empezó con un análisis (Heidrich y Redondo, 2011), que tenía como objetivo confirmar o contrariar la percepción inicial de que mismo que el uso de representaciones digitales ya conste del cotidiano de las escuelas de arquitectura, sigue el predominio de las representaciones planas y estáticas utilizadas desde siempre. Para esto, fue elegido un universo limitado a una escuela de arquitectura en Brasil, la Faculdade de Arquitetura e Urbanismo (FAUrb) de la Universidade Federal de Pelotas (UFPel), donde fueron aplicados cuestionarios a profesores y alumnos.

Los datos obtenidos identificaron y confirmaron el predominio de representaciones planas y estáticas como medio de representación y análisis de proyecto arquitectónico, mismo con la predominancia de la utilización de representaciones desarrolladas en medio digital. Estos datos originaron un estudio, a que se refiere este texto, con objetivo de proponer el uso de representaciones digitales con características de interactividad y movimiento en el análisis visual de proyectos arquitectónicos desarrollados en medio académico.

De este modo, el presente texto propone dos métodos para el desarrollo de representaciones con interactividad y movimiento y describe una investigación hecha con algunos estudiantes de arquitectura de la escuela mencionada, para averiguar primeramente la viabilidad de utilización de las representaciones propuestas.

\section{Experiencia Visual Arquitectónica}

Para Dondis (2007) “expandir la capacidad de ver significa expandir nuestra capacidad de comprender un mensaje visual y, lo que aún es más importante, de elaborar un mensaje visual.” (p.20). Según el autor, la visión incluye algo más que el hecho físico de ver, o de que se nos enseñe algo, "es parte integrante del proceso de comunicación que engloba todas las consideraciones de las bellas artes, las artes aplicadas, la expresión subjetiva y la respuesta a un propósito funcional"(p.20).

Por lo tanto, Dondis (2007) concluye que "ver un objeto proporciona en ocasiones un conocimiento suficiente para evaluarlo y comprenderlo" (p.26). Para el autor, "este carácter de la observación no solo sirve como artificio que nos capacita para aprender, sino también como nuestro vinculo más estrecho con la realidad de nuestro entorno." (p.26)

De este modo, según comenta Norberg-Schulz (1998), "es natural definir la verdadera experiencia arquitectónica como la percepción de la totalidad arquitectónica. Así pues, la experiencia arquitectónica debería corresponder a una descripción relevante de la obra en cuestión". (p.125).

Así, parece evidente que, para un análisis de la experiencia visual arquitectónica consistente, debemos ter el movimiento asociado a la percepción visual, o sea, es necesario movernos constantemente de un lado a otro para que tal percepción sea facilitada, esto se tratando de una construcción real o mismo de una representación gráfica. 


\section{Representación Gráfica Arquitectónica}

La enseńanza del proyecto arquitectónico es una modalidad de simulación, en la cual su análisis es hecho didácticamente con la utilización de representaciones gráficas. Sin embargo, según comenta Sainz (1990), "la representación del espacio arquitectónico se ve restringida por las propiedades del medio gráfico en que se desenvuelve" (p. 30). Cuestión relacionada no solamente con los recursos tecnológicos utilizados, pero principalmente con los sistemas gráficos establecidos como base de las representaciones utilizadas en el análisis de los proyectos.

En este sentido, Bloomer (1982) observa que "cualquier arquitectura es un estímulo potencial del movimiento, sea este real, o imaginado" (p.72). Según el autor, una edificación "es siempre un estimulante para la acción, un escenario en el que tienen lugar la interacción y el movimiento" (p.72). Sainz (1990) también comenta que: "la experiencia de la arquitectura en general, y del espacio arquitectónico en particular, se caracteriza por ser dinámica, continua y variable". (p.69)

Por su parte, según demuestra los datos de Heidrich y Redondo (2011), los estudiantes de arquitectura, del contexto de este estudio, utilizan a diario representaciones que tienen atributos contrarios a estas características de la experiencia arquitectónica, o sea, son bidimensionales y estáticas.

Así que este estudio buscó investigar la posibilidad de utilización de ambientes interactivos y realidad aumentada como representación de la experiencia arquitectónica, para el análisis visual de los espacios arquitectónicos propuestos por proyecto de estudiantes de arquitectura.

\section{Representación del Análisis Visual con Interacción $Y$ Movimiento}

El uso de medios representativos con las características de interacción y movimiento, normalmente significan la necesidad de conocimientos específicos de informática, debido la necesidad de utilizar software llamados de game engine, o aún algún lenguaje de programación para definir algún tipo de interacción, lo que también, normalmente, significan un grande tiempo de dedicación al desarrollo de este tipo de representación.

Debido a esto, este estudio empezó con la búsqueda de medios de representación con interacción y movimiento que utilicen conocimientos y productos gráficos ya normalmente desarrollados por los estudiantes, con el objetivo de que la utilización de estos medios representativos no exigiese acrecimos de dedicación en la técnica de representación, desviando el alumno del desarrollo específico del proyecto.

De este modo, fueron buscadas herramientas que tuviesen su utilización asociada a modelos tridimensionales digitales desarrollados en Sketchup, software de modelado digital ya normalmente utilizado por los alumnos.

Así, se propuso el uso de herramientas digitales específicas para dos tipos de representación: la experiencia visual en los espacios propuestos, y la visualización del objeto arquitectónico propuesto en su contexto de implantación.

\section{Representación de la Experiencia Visual en los Espacios Propuestos}

Para este tipo de representación la herramienta de utilización propuesta por este estudio fue el software Walkabout3D, que a pesar de tener una visualización del modelo digital en un tipo de renderización no fotorrealista (Figura 1), tiene todas las interactividades de un ambiente en realidad virtual no inmersiva, y además, permite una visualización en tercera persona, o sea, con la visualización de un elemento (avatar) que representa el observador dentro del modelo digital, lo que facilita la referencia de proporción y escala.

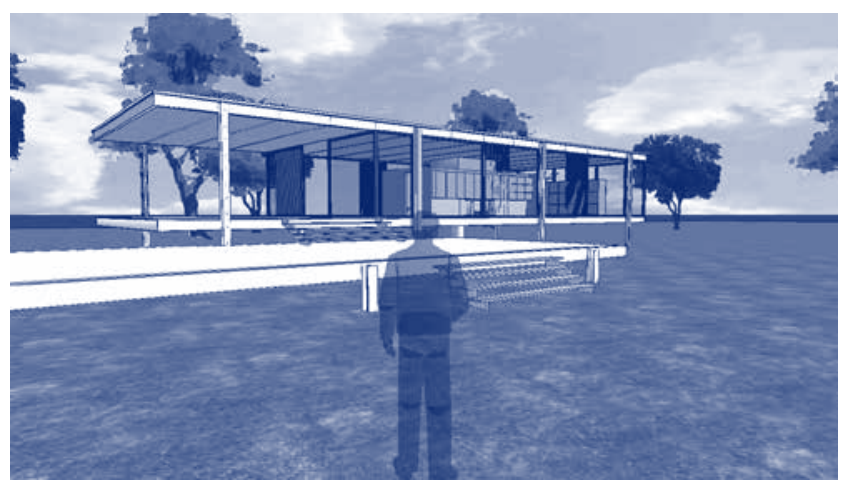

Figura 1: Visualización del modelo digital en el Walkabout3D.

Así que su método de uso consiste en tres etapas:

1. Desarrollo del modelo tridimensional digital de los espacios propuestos;

2. Conversión del modelo tridimensional digital en archivo de ambiente interactivo;

3. Manipulación de la visualización del modelo como ambiente interactivo.

En la primera etapa, la utilización de la herramienta propuesta permite el uso directo del archivo en formato .SKP (original del Sketchup) como ambiente interactivo (Figura 2), entonces, ninguna alteración necesita ser hecha en lo que los alumnos ya normalmente hacen.
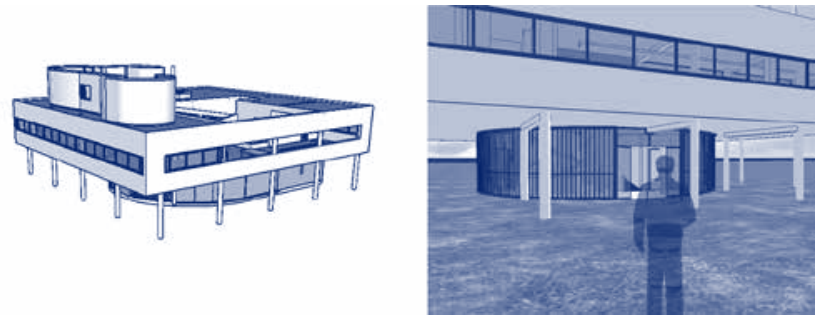

Figura 2: Modelo del Sketchup y su visualización como ambiente interactivo.

En la segunda etapa, como el software propuesto necesita una licencia paga y su uso se detiene apenas a abrir el archivo desarrollado por el estudiante y exportar para otro formato, el desarrollo de este tipo de representación en esta etapa, para los alumnos, consistiría en utilizar un ordenador con el software licenciado instalado, y hacer la conversión del modelo digital en ambiente interactivo. 
La etapa final, de manipulación del modelo interactivo, consiste en la utilización de un software de visualización de uso libre, así que los estudiantes pueden hacerla en sus propios ordenadores.

Como el método de utilización de la representación propuesta para los alumnos consiste básicamente en desarrollar un modelado digital, que ya normalmente lo hacen, y visualizarlo de forma distinta, el estudio buscó analizar la viabilidad de utilización de esta representación, a partir del análisis de la manipulación hecha por los estudiantes sobre un ejemplo de modelo interactivo.

\section{Representación del Objeto Arquitectónico \\ Propuesto en su Contexto de Implantación}

Para este tipo de representación, el estudio propone la utilización de la técnica de visualización de modelos tridimensionales digitales en realidad aumentada, que permite hacer algo como un fotomontaje en tiempo real. Se considera esta técnica una mejoría a la técnica de simple fotomontaje, porque conforme comenta Rasmussen (1998) "alguien que tenga visto un local primeramente en una foto y después lo tenga visitado, sabe como la realidad es bien distinta. Se puede sentir la atmosfera al rededor y ya no se está limitado al ángulo de visión de donde fue sacada la foto”. Así la presencia del alumno en el contexto de implantación del objeto arquitectónico puede colaborar con el análisis de su proyecto.

La herramienta elegida para utilización de esta técnica fue el Armedia for Sketchup, que permite la georreferenciación del modelo digital, lo que posibilita la visualización del modelo digital en realidad aumentada sin ningún tipo de marcador y en el local planeado por el proyecto.

Así que su método de uso consiste en tres etapas:

1. Desarrollo del modelo tridimensional digital georreferenciado del objeto arquitectónico propuesto;

2. Conversión del modelo tridimensional digital en archivo de visualización en realidad aumentada;

3. Manipulación de la visualización en realidad aumentada del modelo digital.

En la primera etapa, como la herramienta propuesta parte del uso directo del archivo en formato.SKP (original del Sketchup), la única alteración necesaria en lo que los alumnos ya normalmente hacen, es la georreferenciación del modelo, o sea, la inclusión de las informaciones de localización geográfica (Figura 3).
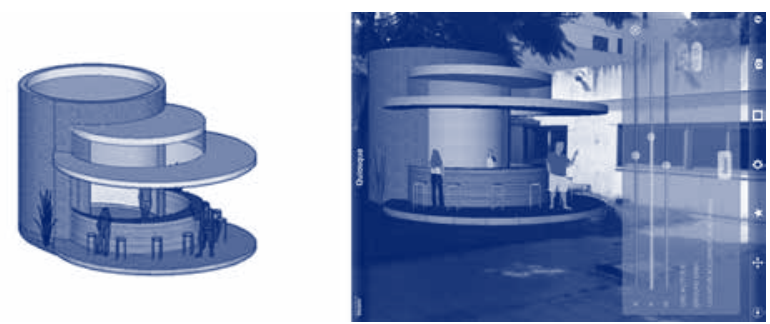

Figura 3: Modelo del Sketchup y su visualización en Realidad Aumentada.

En la segunda etapa, como el uso del Armedia for Sketchup necesita una licencia paga y su uso consiste apenas en abrir el archivo desarrollado por el estudiante y exportar para otro formato, el desarrollo de este tipo de representación en esta etapa, para los alumnos, consistiría en utilizar un ordenador con el software licenciado instalado, y hacer la conversión del modelo digital para el modelo de visualización en realidad aumentada.

La etapa de manipulación de la visualización en realidad aumentada del modelo digital consiste en la utilización de un aplicativo de visualización gratuito para tablets o smartphones, así que los estudiantes necesitan apenas tener acceso a este tipo de equipamiento y poner el archivo de realidad aumentada del modelo en el equipamiento a ser utilizado.

Como el método de utilización de esta representación propuesta para los alumnos consiste en desarrollar un modelado digital que ya normalmente lo hacen, y visualizarlo de forma distinta, el estudio buscó analizar la viabilidad de utilización de esta representación, a partir del análisis de la manipulación, hecha por los estudiantes, de un ejemplo de modelo digital preparado para este tipo de visualización.

\section{Ejemplos de las Representaciones Propuestas}

Para el desarrollo de los ejemplos de las representaciones fueron utilizados tres modelos tridimensionales modelados en Sketchup, dos para verificación del uso del ambiente interactivo (Farnsworth House del arquitecto Mies van der Rohe y Ville Saboye del arquitecto Le Corbusier) y uno para la verificación del uso de la simulación del objeto arquitectónico en tamaño natural, georreferenciado y en su contexto de implantación (un quiosco, elegido para facilitar la visualización del ejemplo, pues tornó posible la visualización en un patio de la escuela donde se hizo el estudio). Todos los modelos fueran obtenidos de un banco de modelos tridimensionales digitales denominado 3D Warehouse (Figura 4).

A partir de estos modelos, fueron utilizadas las herramientas propuestas y hechas las conversiones necesarias. De este modo, los modelos de la Farnsworth House y de la Ville Saboye fueron convertidos en modelos interactivos, y el modelo del quiosco fue convertido en modelo georreferenciado, con informaciones geográficas del patio de la escuela, y para visualización en realidad aumentada. Estos tres modelos fueron entonces utilizados como ejemplos para el test con los alumnos de la manipulación y visualización de las representaciones propuestas.

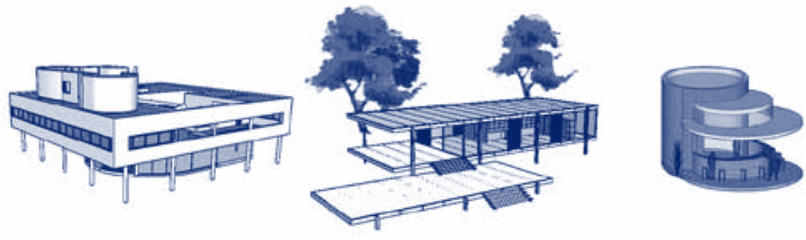

Figura 4: Modelos del Sketchup utilizados como ejemplos de las representaciones propuestas.

\section{Análisis de la Viabilidad de Utilización de las Representaciones Propuestas}

Para el análisis, fue formulado un cuestionario para colectar las opiniones de los estudiantes acerca de la manipulación y del uso de las representaciones. Antes de contestaren las preguntas del cuestionario, todos los veinte uno alumnos que participaron del 
estudio, visualizaron y manipularon individualmente los tres tipos de representación.

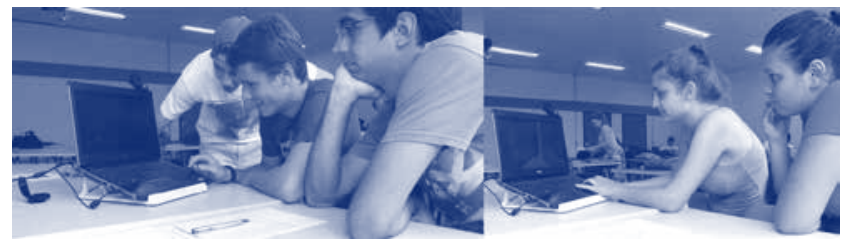

Figura 5: Alumnos utilizando el ejemplo de representación como ambiente interactivo.

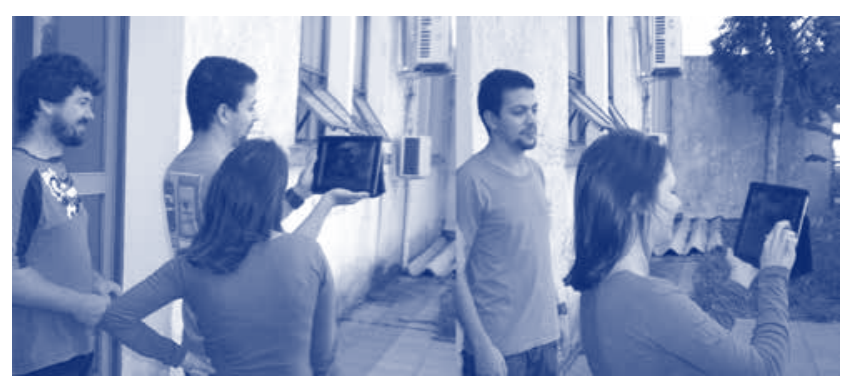

Figura 6: Alumnos utilizando el ejemplo de representación del objeto en su contexto de implantación.

Los datos colectados con las respuestas de los estudiantes dicen, sobre la manipulación de las representaciones propuestas, que: $81 \%$ de los alumnos las consideraron simple y de rápido aprendizaje; y el 19\% de los alumnos las consideraron complejas, pero de rápido aprendizaje.

Cuanto al uso de las representaciones propuestas: $100 \%$ de los alumnos las consideraron útiles, y el 70\% consideraron que cualificaría la visualización del proyecto. Específicamente sobre cada representación: $88 \%$ gustarían de utilizar la simulación georreferenciada y contextualizada de sus proyectos y el mismo porcentaje $(88 \%)$ gustarían de representar sus proyectos con el ambiente interactivo.

\section{Conclusiones}

El objetivo de este estudio fue hacer una investigación preliminar de introducción de técnicas de representación que tornen el análisis visual de los proyectos arquitectónicos desarrollados en medio académico más cerca de la experiencia arquitectónica real.
En este sentido, los datos obtenidos con el presente estudio señalan una posibilidad de introducción, en lo que se dice respecto a la utilización y manipulación por parte de los estudiantes, de los dos tipos de representación propuestos para el análisis visual de proyectos arquitectónicos académicos.

Sin embargo, además de esta posibilidad de uso, lo que este estudio demostró principalmente fue la facilidad de obtención de las representaciones con interactividad y movimiento, pues el desarrollo de las representaciones propuestas está basado solamente en la existencia del modelo tridimensional digital en Sketchup que los estudiantes ya normalmente desarrollan. Así que este estudio expone una necesidad de observación y tal vez revisión en las representaciones de proyectos arquitectónicos desarrollados a menudo en el medio académico.

\section{Agradecimientos}

Los autores agradecen a todos los estudiantes que participaron voluntariamente en el estudio

\section{Referencias}

Bloomer, K. (1982). Cuerpo, memoria y arquitectura. Título original: Body, Memory, and Architecture. Traducido por: María Teresa Muñoz Jiménez. Madrid: Blume Ediciones.

Dondis, D. (2007). La sintaxis de la imagen: Introducción al alfabeto visual. Título original: A Primer of Visual Literacy (1973). Versión castellana de Justo G. Beramendi. 19a edición. Barcelona: Editorial Gustavo Gili.

Heidrich, F.; Redondo, E. (2011). El uso del Medio Digital y el Predominio de las Representaciones Planas y Estáticas. In: IX International Conference on Graphics Engineering for Arts and Design, 2011, Rio de Janeiro. Expressão Gráfica Conexóes entre Ciência, Arte e Tecnologia. Rio de Janeiro: UFRJ.

Norberg-Schulz, C. (1998). Intenciones en Arquitectura. Título original: Intensjoner i arkitekture. Versión castellana de Jorge Sainz Avia y Fernando Gonzalez Valderrama (1967). 2 2a edición. Barcelona: Editorial Gustavo Gili.

Rasmussen, S. (1998). Arquitetura Vivenciada. Titulo original: Experiencing Architecture. Tradução: Álvaro Cabral. 2a ed. São Paulo: Martins Fontes.

Sainz, J. (2005) El Dibujo de Arquitectura: Teoría e historia de un lenguaje gráfico. (Primera Edición, 1990). Madrid: Editorial Reverté. 